\title{
Necrosis grasa del recién nacido: A propósito de un caso
}

\author{
ESTER AVAYÚ H. ${ }^{1}$, CAROLYN RODRÍGUEZ A. ${ }^{2}$, \\ XIMENA WORTSMAN C. ${ }^{3}$, YAMILE CORREDOIRA S. ${ }^{4}$, \\ DAFNE SERMAN V. ${ }^{5}$ GABRIELA STRAUCH B. ${ }^{6}$, LORETO FARÍAS S. ${ }^{7 *}$ \\ 1. Dermatóloga, Servicio de Dermatología, CRS Cordillera. \\ 2. Dermatóloga, Servicio de Dermatología, CRS Cordillera, Universidad de Chile. \\ 3. Radióloga, Servicio de Radiología, Clínica Server. \\ 4. Anatomopatóloga, Servicio de Anatomía-Patológica, Hospital Luis Tisné, Depto. Patología Campus Centro, \\ Facultad de Medicina, Universidad de Chile. \\ 5. Pediatra, Servicio de Pediatría, CRS Cordillera. \\ 6. Becada Dermatología, Universidad de Chile. \\ 7. Interna VII año Medicina, Universidad de Chile.
}

\begin{abstract}
Newborn fat necrosis: Case-report

Newborn fat necrosis is an unfrequent disease that compromises the adipose tissue and develops during the first weeks of life. It is characterized by reddish-purple nodules and/or plaques with a hard elastic consistency, isolated or multiple, localized at the back, shoulders, buttocks, arms, thighs and chin. They evolve spontaneously to regression and unfrequently develop complications, being hypercalcemia the most observed. The pathogenesis of newborn fat necrosis is unknown, although certain risk factors for its development have been identified. Case-report: A 36 weeks preterm male newborn with a clinical picture compatible with fat necrosis. The diagnosis was made according to the clinical findings and his perinatal history, confirming it with pathology. The patient evolution was complete improvement and regression of the lesions, without complications.

(Key words: Newborn, fat necrosis, hypercalcemia, perinatal).

Rev Chil Pediatr 2009; 80 (1): 60-64
\end{abstract}

\section{RESUMEN}

La necrosis grasa del recién nacido (NGRN) es una rara enfermedad que afecta al tejido adiposo y que se desarrolla durante las primeras semanas de vida. Se caracteriza por nódulos y/o placas eritemato-violáceas, de consistencia duro-elástica, únicas o múltiples, en dorso, hombros, glúteos, brazos, muslos y mejillas, que evolucionan espontáneamente hacia la regresión. Si bien pueden desarrollarse complicaciones, éstas son infrecuentes, siendo la más importante la hipercalcemia. La patogenia de la NGRN es desconocida,

Trabajo recibido el 12 de octubre de 2008, devuelto para corregir el 20 de noviembre de 2008, segunda versión el 05 de diciembre de 2008, aceptado para publicación el 18 de diciembre de 2008.

Correspondencia a:

Ester Avayú $\mathrm{H}$.

E-mail: avayu_cl@hotmail.com 
aunque se han identificado factores predisponentes para su desarrollo. Presentamos el caso de un recién nacido de pretérmino de 36 semanas, sexo masculino, que presentó un cuadro clínico compatible con NGRN. El diagnóstico se sospechó en base a la clínica, a los antecedentes perinatales y se confirmó con el estudio histopatológico. El paciente evolucionó satisfactoriamente con regresión de las lesiones y sin presentar complicaciones hasta la fecha.

(Palabras clave: Necrosis grasa, hipercalcemia, recién nacido, perinatal).

Rev Chil Pediatr 2009; 80 (1): 60-64

\section{Introducción}

La necrosis grasa del recién nacido (NGRN) es una rara enfermedad que afecta al tejido adiposo y que se desarrolla durante las primeras semanas de vida, en recién nacidos de término o post-término ${ }^{1-2}$.

Las lesiones aparecen entre la primera y la sexta semana de vida con edema y eritema, que evolucionan a nódulos y placas eritematovioláceas en dorso, hombros, glúteos, brazos, muslos y mejillas. Son de consistencia duroelástica y pueden ser únicas o múltiples. Suelen ser asintomáticas, aunque puede existir sensibilidad local que conduce al neonato al llanto cuando es manipulado ${ }^{3}$.

Su patogenia es desconocida, pero la principal hipótesis plantea que condiciones locales o sistémicas pueden inducir hipoxemia subcutánea y necrosis de adipocitos ${ }^{1-2}$.

Se han identificado factores predisponentes para el desarrollo de esta enfermedad:

Maternos: Diabetes gestacional, preeclampsia, uso de bloqueadores de calcio o consumo de cocaína durante el embarazo.

Complicaciones del parto: Hipoxemia, hipotermia, infecciones y trauma cutáneo.

Neonatales: Anemia o trombocitosis ${ }^{4}$.

El diagnóstico se realiza en base al cuadro clínico y al estudio histopatológico, el cual evidencia necrosis grasa con un infiltrado granulomatoso compuesto de linfocitos, histiocitos, células gigantes tipo cuerpo extraño y depósito de cristales de colesterol ${ }^{5-7}$.

La ecotomografia doppler color es un examen no invasivo que permite apoyar al diagnostico clínico, ya que existen signos sugerentes de la presencia de necrosis grasa, tales como el aumento de grosor y ecogenicidad del tejido celular subcutáneo, así como también la pre- sencia de focos anecogénicos de licuefacción del tejido adiposo.

La NGRN generalmente sigue un curso benigno, con completa regresión de las lesiones en pocas semanas (en algunos casos puede permanecer una leve atrofia de la piel luego de la resolución del cuadro). Sin embargo, pueden presentarse complicaciones graves como trombocitopenia, hipoglicemia, hipertrigliceridemia e hipercalcemia ${ }^{8}$. Esta última es la complicación más frecuente y seria; aparece cuando la necrosis grasa comienza a resolverse, manifestándose con letargia, irritabilidad, hipotonía, vómitos, poliuria, polidipsia, deshidratación, constipación y mal progreso ponderal ${ }^{9-11}$.

Debido a que la NGRN es un cuadro autolimitado, el manejo está enfocado a la pesquisa y tratamiento de las posibles complicaciones, para esto es necesario solicitar hemograma, glicemia, perfil lipídico y calcemia. La hipercalcemia puede aparecer hasta 1-6 meses luego del inicio de las lesiones en piel, por lo que se recomienda controlar calcemia semanal o bisemanalmente al menos durante seis meses ${ }^{8}$.

\section{Caso Clínico}

Recién nacido de pretérmino, 36 semanas, grande para la edad gestacional, sexo masculino, hijo de madre con antecedentes de hipertensión arterial crónica y diabetes gestacional. Nació por cesárea por presentar registro basal no estresante (RBNS) no reactivo, obteniendo un APGAR 4-8. Evolucionó con apneas, desaturación y bradicardia, requiriendo ventilación a presión positiva y ventilación mecánica por 24 horas. A las 2 horas de vida presentó hipoglicemia asociada a cuadro convulsivo, evolucionando con hipotonía leve, que se interpretó 
como Encefalopatía Hipóxico Isquémica, iniciándose fenobarbital. Se realizan hemograma, calcemia, pruebas hepáticas, ecografía cerebral y ecocardiografía que resultaron normales. Paciente evolucionó favorablemente por lo que fue dado de alta al décimo día de vida.

Madre refiere que desde el cuarto día de vida, comienzan a aparecer en la piel de dorso y mejilla zonas de consistencia aumentada que iban en crecimiento. Al examen se observaron múltiples nódulos subcutáneos de consistencia gomosa y superficie eritemato-violácea pálida, entre 2 a $5 \mathrm{~cm}$. en su diámetro mayor, bien delimitados y fijos a planos profundos en dorso, brazos y mejilla derecha (figura 1 y 2).

Se solicitó ecografía de partes blandas que mostró paniculitis con focos de necrosis grasa (figura 3). Se realizó biopsia de un nódulo dorsal (figura 4) que evidenció paniculitis lobular sin vasculitis, predominantemente histiocitaria, con cristales lipídicos intracitoplasmáticos histiocitarios y adipocíticos, consistente con necrosis grasa subcutánea del recién nacido.

Paciente actualmente de 1 mes de vida, en
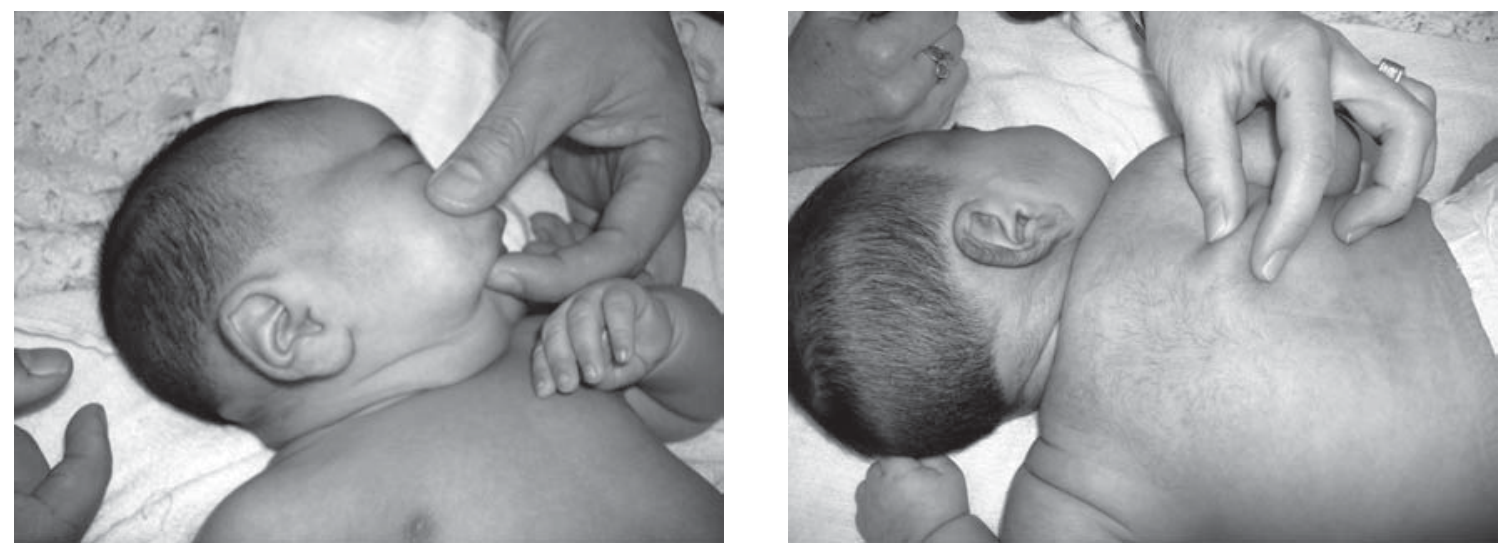

Figura 1 y 2. Recién nacido con nódulos subcutáneos en mejilla y dorso, de consistencia gomosa y bien delimitados. Lesiones son más palpables que visibles.

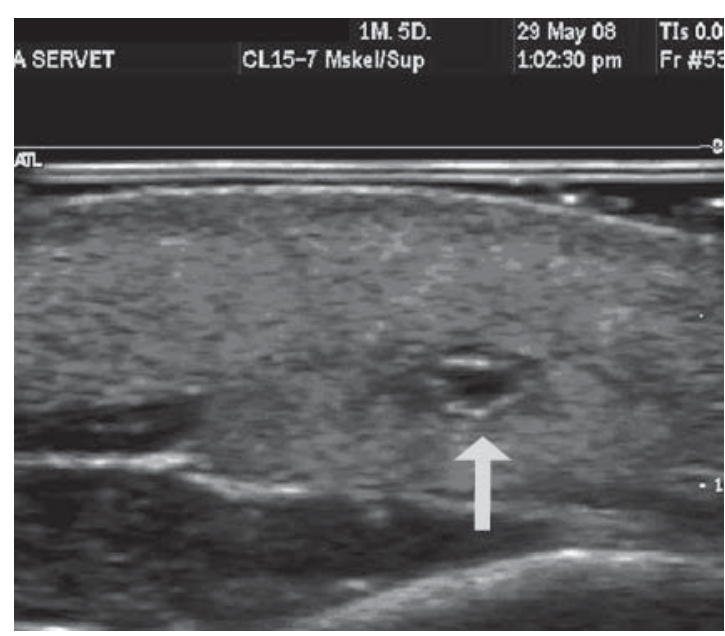

Figura 3. Engrosamiento y aumento de ecogenicidad del tejido celular subcutáneo asociado a una imagen anecogénica redondeada (flecha) sugerente de foco de necrosis grasa en el dorso del paciente.

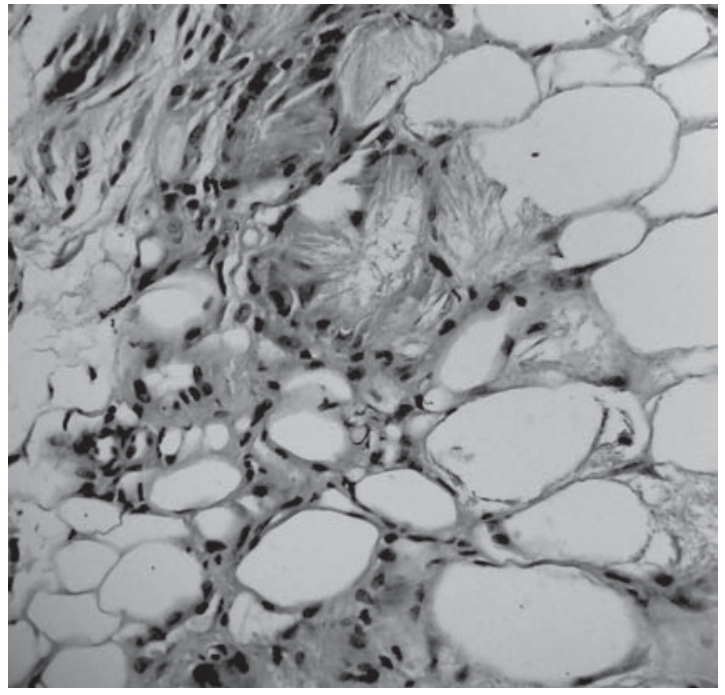

Figura 4. Necrosis de adipocitos e infiltrado linfohistiocitario con células gigantes multinucleadas. 
buenas condiciones generales, con lesiones disminuyendo de tamaño y de coloración menos intensa. El nódulo de la mejilla derecha ha presentado una menor regresión en relación a los otros. Se ha realizado control seriado de calcemia, glicemia, hemograma y perfil lipídico, los cuales se ha mantenido en rangos normales.

\section{Discusión}

La NGRN es una afección poco frecuente, de curso benigno y autolimitado que afecta en las primeras semanas de vida a recién nacidos de término y post-término ${ }^{3}$. Si bien, nuestro paciente fue un recién nacido de pretérmino de 36 semanas, presentaba algunos factores predisponentes para el desarrollo de la NGRN, tales como diabetes gestacional materna e hipoxia del recién nacido durante el parto.

Las lesiones comenzaron a manifestarse dentro de la primera semana de vida como las que característicamente se describen en este cuadro: nódulos eritemato-violáceos sobre dorso, brazos y mejillas.

El diagnóstico se sospechó en base a los antecedentes perinatales y al cuadro clínico, se realizó una ecografía doppler color que fue compatible, confirmándose con el estudio anatomopatológico, que demostró una paniculitis sin vasculitis consistente con necrosis grasa del recién nacido.

El principal diagnóstico diferencial de la NGRN es el esclerema neonatorum (tabla 1), el cual aparece en recién nacidos de pretérmino, desnutridos y enfermos (por ejemplo portadores de cardiopatías), presentando una rápida induración difusa del tejido subcutáneo, que respeta palmas, plantas y genitales ${ }^{8}$. La piel se presenta fría, pálida, de consistencia gomosa, con zonas de decoloración moteada La combinación de kalemia elevada con niveles de $\mathrm{CO}_{2}$ disminuidos se ha asociado a desenlaces fatales. La muerte, habitualmente por septicemia, ocurre en aproximadamente $75 \%$ de los ca$\operatorname{sos}^{12}$. Otros diagnósticos que se deben considerar en un neonato con alteraciones del tejido subcutáneo son: infección por citomegalovirus, linfedema congénito, fibromatosis y hemangioma $^{13}$.

Pese a que la NGRN tiene un curso benigno, en algunas ocasiones se pueden desarrollar complicaciones, tales como trombocitopenia, hipoglicemia, hipertrigliceridemia e hipercalcemia $^{8}$. La hipercalcemia es una complicación rara que aparece entre la primera y sexta semana luego de las lesiones, y se acompaña de inapetencia, hipotonía, vómitos, irritabilidad y mal progreso ponderal ${ }^{3}$. Su patogénesis aún es desconocida, pero se postulan diversas hipótesis al respecto. La primera propone que existiría liberación de calcio desde los nódulos en resolución, sin embargo la mayoría de los casos no desarrollan hipercalcemia ni tienen depósito de calcio en las lesiones ${ }^{14}$. La segunda postula que niveles elevados de paratohormona y prostaglandina $\mathrm{E}_{2}$ estimularían la reabsorción ósea ósea, pero la mayoría de los pacientes tienen estos niveles en rango normal ${ }^{9}$. La tercera, y la más aceptada actualmente, propone que existiría un aumento de 1,25 Dihidroxivitamina $\mathrm{D}$, secretada desde los granulomas de los focos de necrosis grasa, estimulando la absorción intestinal de calcio ${ }^{15}$.

Tabla 1. Cuadro comparativo entre Necrosis Grasa y su principal diagnóstico b diferencial, Esclerema neonatorum

\begin{tabular}{lll}
\hline & Necrosis grasa & Esclerema neonatorum \\
\hline Recién nacido & Término o post-término & Pretérmino \\
Antecedentes & $\begin{array}{l}\text { Factores de riesgo maternos, del parto o } \\
\text { neonatales }\end{array}$ & Recién nacido enfermo y desnutrido \\
Lesiones & $\begin{array}{l}\text { Nódulos y/o placas eritemato-violáceas, únicas } \\
\text { o múltiples } \\
\text { Alteraciones bioquímicas } \\
\text { asociadas }\end{array}$ & Hipercalcemia, Trombocitopenia, Hipoglicemia, \\
Pronóstico & Hipertrigliceridemia & Hiperkalemia Bajos niveles de $\mathrm{CO}_{2}$ \\
\hline
\end{tabular}


Afortunadamente, nuestro paciente ha presentado hasta le fecha una evolución favorable, con resolución espontánea de las lesiones y sin presentar complicaciones, las cuales se controlan semanalmente solicitando hemograma, glicemia y calcemia.

Es importante recordar que a todos los neonatos con NGRN se les debe realizar seguimiento con calcemias por al menos 6 meses, ya que la hipercalcemia puede aparecer desde el primer al sexto mes del inicio del cuadro ${ }^{8}$. En caso de presentarse, el tratamiento se basa en el uso de corticoides endovenosos $(2 \mathrm{mg} / \mathrm{kg}$ cada 6 horas), hidratación endovenosa al doble de sus necesidades basales y furosemida a dosis de $1 \mathrm{mg} / \mathrm{kg}$ cada 6 horas para forzar la eliminación renal de calcio ${ }^{8,10}$.

\section{Referencias}

1.- Burden AD, Krafchik BR: Subcutaneous fat necrosis of the newborn: a review of 11 cases. Pediatr Dermatol 1999; 16: 3847.

2.- Mahé E, Descamps V, Belaých S, Crickx B: La cytostéatonécrose du nouveau-né. Presse Med 2002; 31: 612-6.

3.- Grández N, Bravo F: Reporte de un caso de necrosis grasa subcutánea del recién nacido. Folia dermatol Perú 2004; 15: 94-7.

4.- Mahé E, Girszyn N, Hadj-Rabia S, Bodemer C, HamelTeillac D, De Prosa Y: Subcutaneous fat necrosis of the newborn: a systematic evaluation of risk factors, clinical manifestations, complications and outcome of
16 children. British Journal of Dermatology 2007; 156: 709-15.

5.- Larralde M: Dermatología Neonatal y Pediátrica. Buenos Aires: Ediciones Médicas; 1995.

6.- Cuervo J: Necrosis grasa subcutánea del recién nacido. Revista del Hospital de Niños de Buenos Aires. 2002; 44: 196.

7.- Bueno J, De Souza S, Bueno L, Albertoni D: Necrose gordurosa do subcutáneo ou adipo necrose do recien nacido. Descricao de um caso. J Pediatr 1994; 70 : 178-80.

8.- Thao Tran J, Sheth A: Complications of Subcutaneous Fat Necrosis of the Newborn: A Case Report and Review of the Literature. Pediatr Dermatol 2003; 20: 257-61.

9.- Sharata H, Postellon DC, Hashimoto K: Subcutaneous fat necrosis, hypercalcemia, and prostaglandin E. Pediatr Dermatol 1995; 12: 43-7.

10.- Norwood-Galloway A, Lebwohl M, Phelps R, Raucher $H$ : Subcutaneous fat necrosis of the newborn with hypercalcemia. J Am Acad Dermatol 1987; 16: 4359.

11.- Lewis HM, Ferryman S, Gatrad AR, Moss C: Subcutaneous fat necrosis of the newborn associated with hypercalcemia. J R Soc Med 1994; 87: 482-3.

12.- Patterson J: Panniculitis.Sclerema Neonatorum. En: Bolognia Dermatology, USA. Editorial Mosby, 2003; 1560-1.

13.- Anderson DR, Narla LD, Dunn NL: Subcutaneous fat necrosis of the newborn. Pediatr Radiol 1999; 29: 794-6.

14.- Lusk R, Greiman M: Subcutaneous fat necrosis in infancy. Otolaryngol Head Neck Surg 1988; 99: 520-3.

15.- Finne PH, Sanderud J, Aksnes L, Bratlid D, Aarskog $D$ : Hypercalcemia with increased and unregulated 1,25-dihydroxyvitamin $\mathrm{D}$ production in a neonate with subcutaneous fat necrosis. J Pediatr 1988; 112: 792-4. 